\title{
Coronary artery disease risk reclassification using an acoustic-based score in view of the new European Society of Cardiology 2019 guidelines on Chronic Coronary Syndromes
}

\author{
S. E. Schmidt ${ }^{1}$ (I) S. Winther ${ }^{2} \cdot$ M. Boettcher ${ }^{2}$
}

Received: 5 December 2019 / Accepted: 9 December 2019 / Published online: 18 December 2019

(c) The Author(s) 2019

\begin{abstract}
In August 2019, ESC published new guidelines on Chronic Coronary Syndromes including a new risk assessment paradigm for estimation of pre-test-probability. The CAD-score is an acoustic-based score for ruling-out of coronary artery disease (CAD). In the current letter to the editor we re-evaluate the re-classification potential the CAD-score in the view of the new guidelines.
\end{abstract}

Keywords Stable coronary artery disease $\cdot$ Heart sounds $\cdot$ Non-invasive testing $\cdot$ Reclassification $\cdot$ Cost-effectiveness

\section{Introduction}

In July 2019 the International Journal of Cardiovascular Imaging published our study about coronary artery disease (CAD) risk reclassification using an acoustic-based score [1]. A key point in the publication is the capability of the CAD-score to reclassify patient from their original pre-testprobability (PTP) to a lower risk class according to the 2013 European Society of Cardiology (ESC) guidelines on the management of stable CAD. In August 2019, ESC published their new guidelines on Chronic Coronary Syndromes [2]. Key changes in the 2019 guidelines related to estimation of PTP were that the threshold defining low risk was lowered from 15 to 5\%, and the introduction of a new PTP score with PTP values considerably lower than the previously recommended score. In addition, the new score now includes dyspnea as a symptom. In the current letter to the editor we have recalculated the $\mathrm{CAD}$-score reclassification outcome according to the new ESC 2019 guidelines.

S. E. Schmidt

sschmidt@hst.aau.dk

1 Department of Health Science and Technology, Aalborg University, Aalborg, Denmark

2 Department of Cardiology, Region Hospital Herning, Herning, Denmark

\section{Methods}

PTP was calculated for all patients referred with suspected CAD [1] using the new PTP table from the ESC 2019 guidelines. Patients with a PTP equal to or below 5\% were classified as low likelihood. We reclassified patients with a PTP above 5\% into the low likelihood group if their CAD-score was less or equal to 20. For two subjects, the new PTP score could not be calculated as they were below 30 years of age.

\section{Results}

The mean PTP estimated from the ECS 2019 guidelines was $16.2 \%$ (SD: $11.5 \%$ ) in the 1671 patients referred for testing due to suspected CAD. 178 (10.7\%) had low likelihood of CAD $(\leq 5 \%)$. Post CAD-score test this number increased to 679 (40.6\%) (Fig. 2). Before testing, 6 (3.4\%) patients with low PTP had significant-CAD, whereas post-reclassification this number increased to $28(4.1 \%)(\mathrm{p}=0.52)$. If the PTP was not taken into consideration and all patients were classified only according to whenever their CAD-score was less or equal to 20,631 patients (37.8\%) were classified as low likelihood and 24 had significant-CAD (3.8\%). 


\section{Discussion}

In this low prevalence population, the new PTP score from the ESC 2019 guidelines classified $10.7 \%$ of patients as low likelihood. Thereby, to avoid unnecessary testing, the need for reclassifying the remaining nearly $90 \%$ of patients, where the majority is not having CAD but non-invasive testing is recommended, is still evident. As in the original study, 4 out of 10 patients becomes classified as low risk after application of the CAD-score scheme. This reclassification did not increase the prevalence of CAD-subjects in the low risk group above the 5\% limit recommended in the ESC 2019 guidelines (from 3.4 to $4.1 \%$ ). Therefore, the relevance and effectives of the CAD-score as a potential rule-out device persists after application of the new ESC 2019 guidelines on Chronic Coronary Syndromes.

\section{Compliance with ethical standards}

Conflict of interest S. E. Schmidt holds significant shares in Acarix, works significantly as Expert Witness for Acarix and received institutional research grants from Acarix. M. Boettcher works as Expert Witness in Acarix and received institutional research grants from Acarix to conduct clinical studies. S. Winter has no conflict of interest.
Open Access This article is licensed under a Creative Commons Attribution 4.0 International License, which permits use, sharing, adaptation, distribution and reproduction in any medium or format, as long as you give appropriate credit to the original author(s) and the source, provide a link to the Creative Commons licence, and indicate if changes were made. The images or other third party material in this article are included in the article's Creative Commons licence, unless indicated otherwise in a credit line to the material. If material is not included in the article's Creative Commons licence and your intended use is not permitted by statutory regulation or exceeds the permitted use, you will need to obtain permission directly from the copyright holder. To view a copy of this licence, visit http://creativecommons.org/licenses/by/4.0/.

\section{References}

1. Schmidt SE et al (2019) Coronary artery disease risk reclassification by a new acoustic-based score. Int J Cardiovasc Imaging 35:2019-2028

2. Knuuti J et al (2019) 2019 ESC Guidelines for the diagnosis and management of chronic coronary syndromes. Eur Heart J. https ://doi.org/10.1093/eurheartj/ehz425

Publisher's Note Springer Nature remains neutral with regard to jurisdictional claims in published maps and institutional affiliations. 